\title{
Seguridad de la exclusión quirúrgica del apéndice auricular izquierdo en pacientes con fibrilación auricular sometidos a cirugía valvular mitral
}

\author{
Dres. María José Arocena, Amparo Fernández, Martín Canessa, \\ Macarena Lorente, Facundo Ríos, Guillermo Agorrody, Ricardo Robaina, \\ Víctor Dayan
}

\section{Resumen}

Introducción: la fibrilación auricular (FA) es la arritmia sostenida más frecuente en la práctica clínica. Asocia un aumento significativo de morbimortalidad. La prevención de fenómenos embólicos es un pilar del tratamiento, basado en gran medida en la anticoagulación oral (ACO). Hay un porcentaje significativo de pacientes que presentan contraindicaciones para ACO, a los cuales se deben ofrecer tratamientos alternativos. La exclusión quirúrgica del apéndice auricular izquierdo (AAI) determina beneficios en tal sentido y se asocia a una menor tasa de eventos neurológicos.

Objetivo: primario, valorar la seguridad de la exclusión quirúrgica del AAI en pacientes con FA sometidos a cirugía valvular mitral; secundario, analizar la incidencia de ataque cerebrovascular (ACV) en dicha población y analizar la sobrevida entre los pacientes con y sin exclusión del AAI.

Método: estudio unicéntrico, analítico, observacional, retrospectivo, comparando exclusión o no del AAI en pacientes con FA sometidos a cirugía cardíaca sobre válvula mitral, entre enero de 2012 y diciembre de 2018. Las variables se obtuvieron de la base de datos institucional. El seguimiento fue telefónico y la sobrevida fue derivada de datos oficiales nacionales.

Resultados: se incluyeron 69 pacientes (en 45 se realizó exclusión del AAI). Como características con diferencias significativas destacan la edad (69,1 $\pm 8,2$ años sin exclusión del AAI; $63,6 \pm 10,3$ años con exclusión del AAI, p=0,026) y el porcentaje de ablación quirúrgica de FA en cada grupo ( $29,2 \%$ sin exclusión del AAI; $68,9 \%$ con exclusión del AAI, p=0,002). Se obtuvo un seguimiento de 33 pacientes, entre los cuales no hubo diferencias significativas en los parámetros considerados. Se evaluó la sobrevida del total de los pacientes incluidos, sin diferencia a largo plazo.

Conclusión: según los datos analizados, la exclusión del AAI es un procedimiento seguro que no agrega complicaciones a la cirugía valvular mitral en pacientes con FA. No fue posible demostrar que la exclusión del AAI reduzca de manera significativa la prevalencia de ACV isquémico a largo plazo, ni modifique la sobrevida.

Palabras clave: $\quad$ FIBRILACIÓN ATRIAL

ACCIDENTE CEREBROVASCULAR

CIRUGÍA CARDÍACA

APÉNDICE ATRIAL

\section{Safety of left atrial appendage exclusion in patients with atrial fibrillation undergoing mitral valve surgery.}

\section{Summary}

Introduction: atrial fibrillation is the most common sustained cardiac arrythmia in clinical practice. It is associated to significant morbimortality. The prevention of embolic episodes is a pillar of atrial fibrillation treatment and is based mainly on anticoagulation. However, there is a significant proportion of patients with contraindications for anticoagula-

Instituto Nacional de Cirugía Cardíaca. Montevideo, Uruguay.

Correspondencia: Dra. María José Arocena Morales. Correo electrónico: majoarocena@gmail.com

Los autores declaran no tener conflictos de intereses.

El presente estudio no tiene fuentes de financiamiento.

Recibido Mar 26, 2020; aceptado Jul 6, 2020. 
tion, to whom alternative treatments must be offered. Surgical exclusion of the left atrial appendage offers benefits in this regard, and is associated to a lower rate of neurological events.

Objective: primary endpoint: to evaluate the safety of the surgical exclusion of the left atrial appendage in patients with atrial fibrillation undergoing mitral valve surgery; secondary endpoint: to analyze the incidence of stroke in this population, and to analyze the survival rate in patients with and without left atrial appendage exclusion.

Method: a single center, analytic, observational, retrospective study, comparing exclusion and no exclusion of the left atrial appendage in patients with atrial fibrillation undergoing mitral valve surgery between January 2012 and December 2018. The variables were obtained from the institutional database. The follow up was telephonic and survival rates were obtained from a national official database.

Results: 69 patients were included ( 45 underwent surgical left atrial appendage exclusion). Statistically significant features between the groups were age (69.1 \pm 8.2 years without left atrial appendage exclusion; $63.6 \pm 10.3$ years with left atrial appendage exclusion, $\mathrm{p}=0.026)$, and the rate of surgical ablation of atrial fibrillation $(29.2 \%$ without exclusion; $68.9 \%$ with exclusion, $\mathrm{p}=0.002$ ). The follow up was carried out in 33 , finding no statistically significant differences between the groups. The survival rate of all patients was analyzed, finding no long term differences.

Conclusion: according to the data analyzed, left atrial appendage exclusion is a safe procedure, and adds no further complications to mitral valve surgery in patients with atrial fibrillation. It was not possible to prove that left atrial appendage exclusion significantly reduces the long term prevalence of ischemic stroke or modifies the survival rate.

Key words:

ATRIAL FIBRILLATION
STROKE
CARDIAC SURGERY
ATRIAL APPENDAGE

\section{Segurança da exclusão cirúrgica do apêndice atrial esquerdo em pacientes com fibrilação atrial submetidos a cirurgia valvar mitral.}

\section{Resumo}

Introdução: a fibrilação atrial é a arritmia sustentada mais frequente na prática clínica. Associa um aumento significativo na morbimortalidade. A prevenção de fenômenos embólicos é um dos pilares do tratamento, amplamente baseado na anticoagulação oral. Existe uma percentagem significativa de pacientes que apresentam contra-indicações, a quem tratamentos alternativos devem ser oferecidos. A exclusão cirúrgica do apêndice atrial esquerdo determina benefícios nesse sentido e está associada a uma menor taxa de eventos neurológicos.

Objetivo: primário: avaliar a segurança da exclusão cirúrgica do apêndice atrial esquerdo em pacientes com fibrilação atrial submetidos a cirurgia valvar mitral; secundário: analisar a incidência de ataque cerebrovascular na referida população e análise de sobrevida em pacientes com e sem exclusão de apêndice atrial esquerdo.

Método: estudo de centro único, analítico, observacional, retrospectivo que comparou a exclusão ou não de apêndice atrial esquerdo em pacientes com fibrilação atrial submetidos a cirurgia cardíaca na válvula mitral, entre janeiro de 2012 e dezembro de 2018. As variáveis foram obtidas no banco de dados institucional. O acompanhamento foi por telefone e a sobrevivência foi obtida a partir de dados nacionais oficiais.

Resultados: 69 pacientes foram incluídos ( 45 foram excluídos da apêndice atrial esquerdo). Como elementos estatisticamente significativos, destacam-se a idade (69,1 \pm 8,2 anos sem exclusão do apêndice atrial esquerdo; 63,6 \pm 10,3 anos com exclusão do apêndice atrial esquerdo, $\mathrm{p}=0,026)$ e a taxa de ablação cirúrgica da fibrilação atrial em cada grupo $(29,2 \%$ sem exclusão; $68,9 \%$ com exclusão, $\mathrm{p}=0,002)$. Foi obtido um seguimento de 33 pacientes, entre os quais não houve diferenças significativas nos parâmetros considerados. A sobrevida de todos os pacientes incluídos foi avaliada, sem diferença na sobrevida a longo prazo.

Conclusão: de acordo com os dados analisados, a exclusão do apêndice atrial esquerdo é um procedimento seguro que não agrega complicações à cirurgia valvar mitral em pacientes com fibrilação atrial. Não foi possível demonstrar que a exclusão da apêndice atrial esquerdo reduz significativamente a prevalência de ataque vascular cerebral isquêmico a longo prazo, nem modifica a sobrevida.

Palavras-chave: FIBRILAÇÃO ATRIAL

ACIDENTE VASCULAR CEREBRAL

CIRURGIA CARDÍACA

APÊNDICE ATRIAL 


\section{Introducción}

La fibrilación auricular (FA) es la arritmia sostenida más frecuente en la práctica clínica, alcanzando una prevalencia de $0,5 \%-2 \%$ en la población general ${ }^{(1)}$, subestimada por la población con FA subclínica, y en aumento por la mayor longevidad y elevada prevalencia de factores predisponentes a su desarrollo. Es una enfermedad progresiva que se asocia con un aumento significativo de la morbimortalidad. En el estudio Framingham $^{(2)}$ se siguieron 5.070 participantes a lo largo de 34 años evaluando el impacto de FA no reumática, hipertensión arterial, cardiopatía isquémica e insuficiencia cardíaca (IC) sobre la incidencia de ataque cerebrovascular (ACV). Se concluye que la FA es un factor de riesgo independiente para ACV (aumento en cinco veces), cobrando mayor relevancia en edades más avanzadas. En pacientes con estenosis mitral de etiología reumática se ha descrito un aumento de 17 veces en el riesgo de $\mathrm{ACV}^{(3)}$. Se asocia también a riesgo incrementado de IC (por 3), así como de demencia e infarto de miocardio (por 2)(1). Aproximadamente el $15 \%$ de los ACV isquémicos están relacionados a la $\mathrm{FA}^{(3)}$ y se caracterizan por ser grandes, múltiples, frecuentemente bilaterales y asociados a altas tasas de mortalidad y discapacidad permanente, todo lo cual lleva a que la prevención de fenómenos embólicos sea un pilar en el tratamiento del paciente con FA. La terapia anticoagulante ha tenido gran impacto sobre la incidencia de eventos tromboembólicos relacionados con la FA, pero se estima que hasta $44 \%$ de los pacientes con FA e indicación de anticoagulación presentan al menos una contraindicación, ya sea relativa o absoluta para recibir dicha medicación a largo plazo, sobre todo vinculada al riesgo hemorrágico ${ }^{(1)}$. Es especialmente en esta población que se deben ofrecer alternativas para la prevención de tromboembolias.

El apéndice auricular izquierdo (AAI) ocupa un rol central en la prevención de tromboembolias, dado que es un sitio prevalente para la formación de trombos asociados a la FA. Se ha descrito que hasta el $90 \%$ de los trombos auriculares se originan en el AAI en pacientes con FA no valvular, y el $60 \%$ en pacientes con patología valvular reumática, fundamentalmente estenosis mitral $^{(3)}$. Incluso en pacientes en ritmo sinusal, el sitio de mayor prevalencia de formación de trombos intracavitarios es el AAI. Es por estos motivos que su exclusión forma parte del arsenal terapéutico de la FA en vistas a disminuir la incidencia de tromboembolias a largo plazo $^{(3)}$, especialmente en pacientes con FA no valvular.

\section{Objetivo}

La hipótesis de trabajo es que la exclusión quirúrgica del AAI es segura al no agregar complicaciones a
¿Qué aporta este estudio al conocimiento actual?

- Un porcentaje significativo de pacientes a quienes se les realiza cirugía sobre la válvula mitral padecen fibrilación auricular, y se debe considerar la exclusión quirúrgica del apéndice auricular izquierdo durante la intervención, como tratamiento asociado para su arritmia.

- Se demostró que a nivel de un centro nacional, la exclusión del apéndice auricular izquierdo fue un procedimiento seguro, sin agregado de complicaciones intra o posoperatorias.

la cirugía valvular mitral, y podría determinar menor incidencia de eventos neurológicos. Objetivo primario: valorar la seguridad de la exclusión quirúrgica del AAI en pacientes con FA sometidos a cirugía valvular mitral. Objetivo secundario: analizar la incidencia de ACV en dicha población, y analizar la sobrevida entre los pacientes con y sin exclusión del AAI asociada a cirugía valvular mitral.

\section{Método}

Estudio unicéntrico, analítico, observacional, retrospectivo, comparando exclusión o no del AAI en pacientes con FA que recibieron cirugía sobre válvula mitral, entre enero de 2012 y diciembre de 2018. Se obtuvieron las variables demográficas e intraoperatorias a partir de la base de datos institucional e historia clínica de los pacientes. Las variables consideradas fueron: edad, sexo, obesidad, FA permanente, clase funcional de NYHA $\geq$ II, hipertensión arterial, diabetes mellitus, ACV/ataque isquémico transitorio preoperatorio, cardiopatía isquémica, tabaquismo actual o pasado, dislipemia, enfermedad renal crónica, escala $\mathrm{CHA}_{2} \mathrm{DS}_{2}$-VASc, escala HAS-BLED, fracción de eyección del ventrículo izquierdo, diámetro y área de aurícula izquierda (AI), patología mitral predominante, tipo de prótesis, porcentaje de cirugías combinadas, tiempos de circulación extracorpórea (CEC) y clampeo de aorta (CA), ablación quirúrgica de FA, sangrado en drenajes de tórax, reintervención, complicaciones quirúrgicas y muerte hospitalaria. Las variables evolutivas se obtuvieron mediante contacto telefónico con los pacientes, y fueron: presencia de FA, anticoagulación oral (ACO) y antecedente de ACV luego de la cirugía.

Para el análisis estadístico se aplicaron frecuencia absoluta y porcentajes para las variables cualitativas, media y desvío para las variables cuantitativas y Kaplan-Meier para las curvas de sobrevida. Se aplicaron para el análisis bivariado de variables 


\begin{tabular}{|c|c|c|c|}
\hline Características demográficas & Sin exclusión de $A A I(n=24)$ & Con exclusión de $A A I(n=45)$ & Valorp \\
\hline Sexo masculino & $50,0 \%(12)$ & $62,2 \%(28)$ & NS \\
\hline Edad (años) & $69,17(8,2)$ & $63,64(10,3)$ & 0,026 \\
\hline Obesidad & $37,5 \%(9)$ & $24,4 \%(11)$ & NS \\
\hline FA permanente & $75,0 \%(18)$ & $84,4 \%(38)$ & NS \\
\hline $\begin{array}{l}\text { Insuficiencia cardíaca clase } \\
\text { funcional NYHA } \geq \text { II }\end{array}$ & $83,3 \%(20)$ & $95,6(43)$ & NS \\
\hline Hipertensión arterial & $83,3 \%(20)$ & $64,4 \%(29)$ & NS \\
\hline Diabetes mellitus & $20,8 \%(5)$ & $33,3 \%(15)$ & NS \\
\hline ACV/AIT preoperatorio & $12,5 \%(3)$ & $6,7 \%(3)$ & NS \\
\hline Cardiopatía isquémica & $16,7 \%(4)$ & $11,1 \%(5)$ & NS \\
\hline Tabaquismo & $4,2 \%$ & $15,6 \%(7)$ & NS \\
\hline Extabaquismo & $29,2 \%(7)$ & $26,7 \%(12)$ & NS \\
\hline Dislipemia & $45,8 \%(11)$ & $51,1 \%(23)$ & NS \\
\hline ERC & $12,5 \%(3)$ & $11,1 \%(5)$ & NS \\
\hline Escala $\mathrm{CHA}_{2} \mathrm{DS}_{2}-$ VASc $\geq 2$ & $95,8 \%(23)$ & $88,9 \%(40)$ & NS \\
\hline HAS-BLED $\geq 3$ & $16,7 \%(4)$ & $15,6 \%(7)$ & NS \\
\hline $\begin{array}{l}\text { Fracción de eyección ventrículo } \\
\text { izquierdo }(\%)\end{array}$ & $53,75(13,6)$ & $48,58(12,6)$ & NS \\
\hline Diámetro AI (mm) & $53,61(7,9)$ & $54,20(8,65)$ & NS \\
\hline Área AI $\left(\mathrm{cm}^{2}\right)$ & $53,32(7,85)$ & $53,2(8,8)$ & NS \\
\hline Estenosis mitral & $16,7 \%(4)$ & $17,8 \%(8)$ & NS \\
\hline
\end{tabular}

cualitativas test de chi cuadrado, para las cuantitativas test de T de Student o Mann-Whitney previa valoración de normalidad por medio de Shapiro Wilk. Se utilizó una significancia de 5\% $(\alpha<0,05)$.

\section{Resultados}

Se incluyeron en el estudio 69 pacientes; en 45 se excluyó el AAI y en 24 no se excluyó. Las características basales de la población no difirieron significativamente entre ambos grupos, como se observa en la tabla 1 , a excepción de la edad $(69,1 \pm 8,2$ años sin exclusión del AAI; $63,6 \pm 10,3$ con exclusión del AAI, $\mathrm{p}=0,026)$. De las variables relacionadas con las características de la cirugía se destaca, como la única con diferencia significativa, el porcentaje de ablación quirúrgica de FA ( $29,2 \%$ sin exclusión del AAI; $68,9 \%$ con exclusión del AAI, $\mathrm{p}=0,002$ ) (tabla 2). Los métodos de exclusión quirúrgica fueron: ligadu- ra interna $14(31,1 \%)$, ligadura externa $7(15,5 \%)$, resección $13(28,8 \%)$ y en $11(24,4 \%)$ no se detalló en la descripción operatoria.

Dentro de las complicaciones quirúrgicas se incluyó taponamiento cardíaco, requerimiento de drenaje pericárdico, sangrado mayor, derrame pleural que requiere toracocentesis, infección de esternotomía, requerimiento de implante de marcapasos definitivo, infarto agudo de miocardio y neumotórax.

Se logró el seguimiento de 33 pacientes (12 sin exclusión del AAI; 21 con exclusión del AAI), que fueron contactados a través de comunicación telefónica (tabla 3). En el resto de los casos no fue posible comunicarse debido a cambio de domicilio, o pérdida del contacto telefónico. No hubo pérdida de datos de defunción, ya que se obtuvieron directamente del Ministerio de Salud Pública.

No hubo diferencias significativas entre ambos grupos en cuanto a la presencia de FA diagnosticada 
Tabla 2. Características de la cirugía, comparación entre ambos grupos de pacientes.

\begin{tabular}{|c|c|c|c|}
\hline Características de la cirugía & Sin exclusión de $A A I(n=24)$ & Con exclusión de $A A I(n=45)$ & Valorp \\
\hline \multicolumn{4}{|l|}{ Prótesis mitral } \\
\hline Biológica & $58,3 \%(14)$ & $62,2 \%(28)$ & NS \\
\hline Mecánica & $33,3 \%(8)$ & $37,8 \%(17)$ & NS \\
\hline Reparación & $8,3 \%(2)$ & $0,0 \%$ & NS \\
\hline Cirugía cardíaca combinada & $62,5 \%(15)$ & $42,2 \%(19)$ & NS \\
\hline Ablación quirúrgica de FA & $29,2 \%(7)$ & $68,9 \%(31)$ & 0,002 \\
\hline Tiempo de CEC (minutos) & $121,88(30,67)$ & $119,20(36,0)$ & NS \\
\hline Tiempo de CA (minutos) & $89,83(28,24)$ & $90,42(30,31)$ & NS \\
\hline $\begin{array}{l}\text { Sangrado en drenajes de } \\
\text { tórax }(\mathrm{ml})\end{array}$ & $763,48(529,5)$ & $766,07(623,7)$ & NS \\
\hline $\begin{array}{l}\text { Reintervención por } \\
\text { sangrado }\end{array}$ & $8,3 \%(2)$ & $6,7 \%(3)$ & NS \\
\hline Complicación quirúrgica & $20,8 \%(5)$ & $22,2 \%(10)$ & NS \\
\hline FA al alta & $45,8 \%(11)$ & $64,4 \%(29)$ & NS \\
\hline Muerte hospitalaria & $16,7 \%(4)$ & $4,4 \%(2)$ & NS \\
\hline
\end{tabular}

AAI: apéndice auricular izquierdo; CA: clampeo aorta; CEC: circulación extracorpórea; FA: fibrilación auricular; NS: no significativo.

Tabla 3. Comparación en la evolución entre ambos grupos de pacientes.

Evolución (n=33) Sin exclusión de $A A I(n=12) \quad$ Con exclusión de $A A I(n=21) \quad$ Valor $p$

FA

$\begin{array}{lccc}\text { Sí } & 41,7 \%(5) & 33,3 \%(7) & \text { NS } \\ \text { No } & 50,0 \%(6) & 38,1 \%(8) & \text { NS } \\ \text { Desconoce } & 8,3 \%(1) & 28,6 \%(6) & \text { NS } \\ \text { ACV isquémico } & 16,7 \%(2) & 9,5 \%(2) & \text { NS } \\ \text { ACO } & 100,0 \%(12) & 76,2 \%(16) & \text { NS }\end{array}$

AAI: apéndice auricular izquierdo; ACO: anticoagulación oral; ACV: ataque cerebrovascular; FA: fibrilación auricular; NS: no significativo.

en la evolución, anticoagulación y diagnóstico de ACV en la evolución. Hubo cuatro pacientes con ACV isquémico, dos en cada grupo. De ellos, solo un paciente no estaba anticoagulado (pertenecía al grupo en que no se excluyó el AAI).

Se evaluó la sobrevida del total de los pacientes incluidos. No hubo diferencias estadísticamente significativas en cuanto a las curvas de sobrevida a largo plazo entre los dos grupos (figura 1), pero se evidenció una tendencia a la mayor sobrevida en el grupo de pacientes en que se realizó exclusión del AAI.

\section{Discusión}

En nuestro estudio observamos que la exclusión del $\mathrm{AAI}$ es un procedimiento seguro, que no se relacio- nó con aumento de los tiempos de CA ni de CEC, volumen de sangrado en drenajes de tórax, tasas de reintervención por sangrado, ni otras complicaciones quirúrgicas.

No existen estudios randomizados que comprueben el beneficio de la exclusión quirúrgica del AAI sobre la prevalencia de ACV, si bien esto se ha observado en metaanálisis y estudios retrospectivos. En nuestro trabajo no fue posible comprobar dicho beneficio, pero sí se reflejó la seguridad del procedimiento.

El AAI tiene características anatómicas, ultraestructurales y fisiológicas distintivas de la cámara de la cual forma parte ${ }^{(3)}$. El AAI consta de una formación sacular ciega, de entre 20-60 mm de longitud y 16-59 mm de diámetro ${ }^{(4)}$ y paredes finas, de 


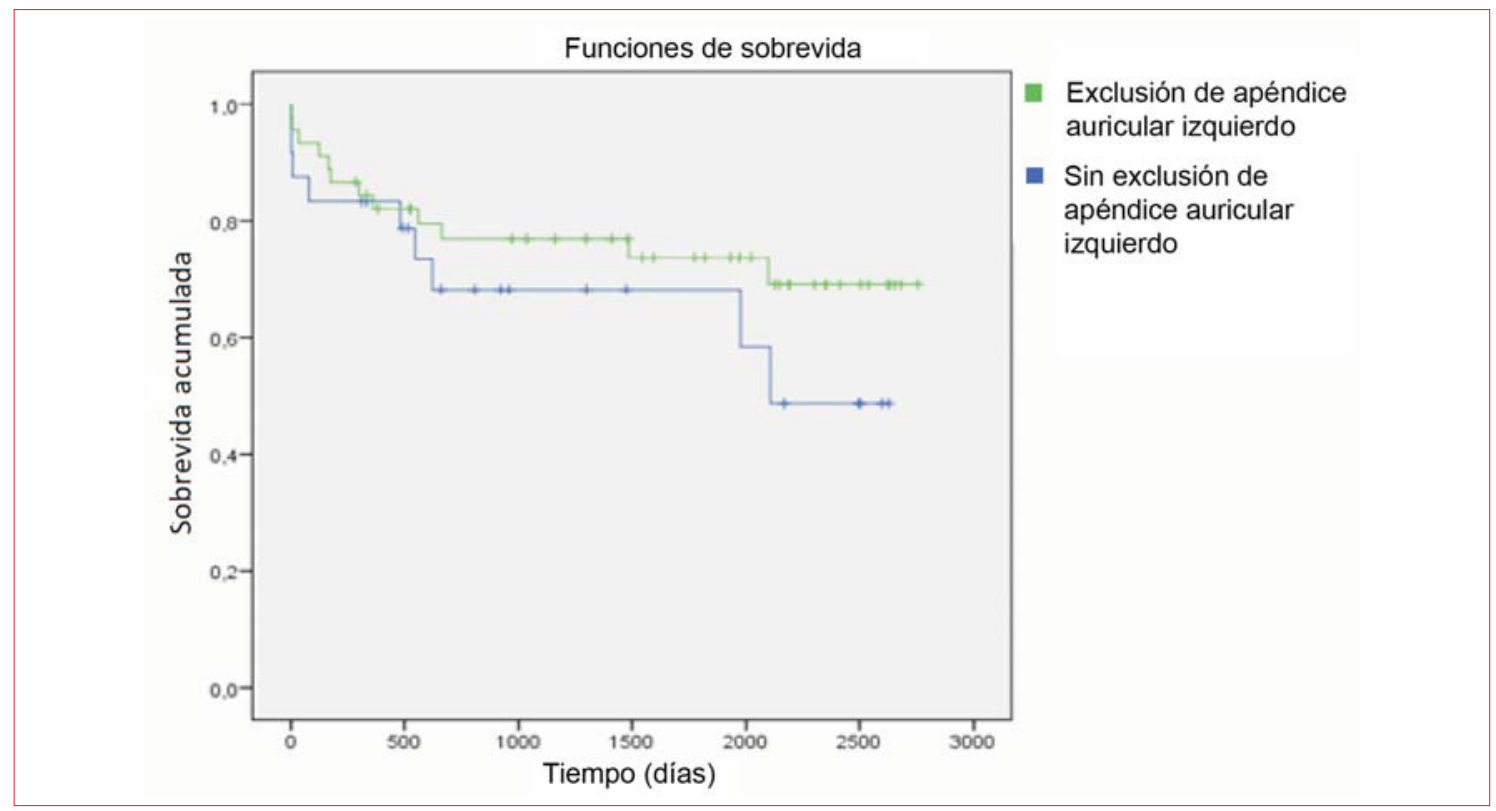

Figura 1. Curvas de sobrevida de Kaplan-Meier.

aproximadamente $1 \mathrm{~mm}$ de espesor, recubierta en su superficie endocárdica con músculos pectíneos que forman trabeculaciones ${ }^{(1)}$. La anatomía es sumamente variable. En el $54 \%$ de la población el AAI es bilobulado y en un tercio es trilobulado, con dichos lóbulos en distintos planos espaciales ${ }^{(1)}$. Existen, a su vez, distintas clasificaciones en cuanto al orificio de entrada al AAI, encontrándose orificios ovales, siendo la forma preponderante (69\%), seguido por orificios en forma de huella (10\%), triangulares $(8 \%)$, en forma de gota $(8 \%)$ y redondos $(6 \%)^{(1)}$.

Las propiedades anatómicas y funcionales del AAI favorecen la estasis sanguínea y formación de trombos en pacientes con FA. El AAI funciona como una cámara descompresiva para la AI durante la sístole y en situaciones de presión elevada intrauricular $^{(2)}$, y su tamaño está supeditado a las condiciones de llenado y al ritmo cardíaco ${ }^{(1)}$. Los factores que favorecen la estasis sanguínea son: auriculomegalia izquierda, presiones de llenado elevadas, contraste espontáneo o "humo" auricular y baja velocidad de flujo en el $\mathrm{AAI}^{(4)}$. En un estudio realizado a partir de análisis de necropsias de pacientes con y sin FA, se encontró que los AAI en pacientes con dicha arritmia eran más voluminosos, con ostia de mayor tamaño y menos ramificaciones que en los pacientes que se encontraban en ritmo sinusal ${ }^{(3)}$. Asimismo, la morfología del AAI incide en el riesgo tromboembólico(1). Se ha postulado que la FA es en sí misma un estado protrombótico, secundario a mecanismos que involucran inflamación, factores de crecimiento, óxido nítrico y sistema renina-angiotensina-aldosterona ${ }^{(4)}$.
Se han desarrollado diversas técnicas para la exclusión del AAI, dentro de las cuales se incluyen abordajes percutáneos y quirúrgicos. Los dispositivos de colocación mediante catéter se basan en tres mecanismos de acción: tipo tapón, en que se obstruye el cuello del AAI sellándolo e impidiendo el pasaje sanguíneo, con epitelización posterior de la superficie en contacto con la cavidad (por ejemplo, Watchman); tipo chupete, en que existe una porción del dispositivo que se introduce en la cavidad del AAI con un disco adicional que sella al AAI (por ejemplo, Amplatzer), y tipo ligadura, que mediante abordaje endo y epicárdico permite excluir al AAI (por ejemplo, Lariat). Los procedimientos percutáneos tienen el objetivo de aislar anatómicamente al AAI, y en caso de la ligadura percutánea, se logra también el aislamiento eléctrico, teniendo como ventaja adicional obviar el contacto de material extraño con el flujo sanguíneo. Raramente existen contraindicaciones anatómicas para la utilización de estos dispositivos, siendo técnicamente factible su utilización en más de $95 \%$ de los pacientes ${ }^{(4)}$.

El tratamiento quirúrgico de la FA incluye la ablación con radiofrecuencia ( $u$ otras fuentes de energía) y la exclusión del AAI, y se realiza generalmente asociada a otra cirugía cardíaca indicada por otra causa en pacientes portadores de FA. La indicación sistemática de la exclusión del AAI durante cirugía cardíaca es discutida, y su grado de recomendación es IIA, nivel de evidencia C, en la Guía de FA 2017 de la Society of Thoracic Surgeons (STS) ${ }^{(5)}$, y IIb, nivel de evidencia B, en la Guía de FA de la European Society of Cardiology (ESC) publica- 
da en $2016^{(6)}$. Los métodos de exclusión quirúrgica incluyen sutura interna, sutura externa, ligadura, colocación externa de grapas y la escisión del AAI. La lesión de la arteria circunfleja en el surco coronario y el sangrado parietal son posibles complicaciones del procedimiento. Luego de la exclusión quirúrgica del AAI existe un porcentaje elevado de exclusión incompleta, que varía de 10\%-80\% en distintas series ${ }^{(1)}$, y que se define como un reservorio tipo pouch $>1 \mathrm{~cm}$ a nivel del remanente apendicular o flujo persistente entre la $\mathrm{AI}$ y el AAI ${ }^{(4)}$. Un estudio randomizado reciente evidenció cierre incompleto en $60 \%$ con método de grapas, $20 \%$ mediante escisión y $13 \%$ mediante ligadura interna ${ }^{(7)}$. La exclusión incompleta del AAI puede incluso favorecer la formación de trombos a través de estasis sanguínea e incrementar el riesgo de fenómenos tromboembólicos en comparación con su exclusión completa; la tasa de tromboembolias en pacientes con exclusión incompleta del AAI que no reciben anticoagulación incrementa en 3-5 veces la estimada a través de la escala $\mathrm{CHA}_{2} \mathrm{DS}_{2}$-VASc.

En un metaanálisis publicado en $2014^{(8)}$, evaluando la eficacia y seguridad de la exclusión quirúrgica del AAI en 3.653 pacientes ( 1.716 con exclusión del AAI y 1.937 sin exclusión), se evidenció menor incidencia de ACV y mortalidad por todas las causas a 30 días con la exclusión del AAI, con tasas comparables de FA posoperatoria y reintervención. En un estudio retrospectivo analizando 75.782 pacientes sometidos a cirugía cardíaca, la exclusión del AAI versus la no exclusión determinó diferencias estadísticamente significativas en cuanto a ACV y mortalidad por todas las causas ${ }^{(9)}$. Un trabajo evaluando la exclusión quirúrgica del AAI en pacientes mayores de 65 años evidenció una menor tasa de reingreso por tromboembolias a tres años en el grupo sometido a exclusión del AAI ${ }^{(10)}$. La Guía de FA de la ESC 2016 recomienda mantener la anticoagulación luego de la exclusión del AAI (indicación I) ${ }^{(6)}$.

El presente trabajo está en línea con los mencionados previamente en cuanto a los parámetros de seguridad evaluados, y si bien no permite concluir acerca del impacto en la sobrevida a largo plazo, con un seguimiento más prolongado y mayor número de casos, podría confirmarse la tendencia de la curva de sobrevida a favor del grupo que recibió exclusión del AAI.

\section{Limitaciones}

Se trata de un estudio retrospectivo que abarca una población limitada. Las técnicas de exclusión del AAI fueron variadas, de acuerdo a la decisión del equipo tratante, y no se realizaron estudios de imagen para comprobar la correcta exclusión del AAI.
Hubo una pérdida considerable de pacientes en el seguimiento por no poderlos ubicar telefónicamente, lo cual representa una de las principales limitaciones del presente trabajo. No contamos con los datos acerca de las causas de muerte, ni si tuvieron eventos tromboembólicos previos al deceso. No hubo pérdida de datos de defunción, ya que se contó con datos oficiales respecto a este evento para el total de los pacientes incluidos en el estudio.

\section{Conclusión}

Según los datos analizados, la exclusión del AAI es un procedimiento seguro que no agrega complicaciones al acto quirúrgico, ni posoperatorias. No se demostró que la exclusión del AAI reduzca de manera significativa la prevalencia de ACV isquémico a largo plazo ni modifique la sobrevida.

Contribución de autores:

María José Arocena, https://orcid.org/0000-0003-40727426 Participación principal en ejecución, análisis, redacción y respuesta a editores.

Amparo Fernández, https://orcid.org/0000-0003-42437844 Recolección de datos.

Martín Canessa, https://orcid.org/0000-0001-8763-2294 Recolección de datos.

Macarena Lorente, https://orcid.org/0000-0003-29746028 Recolección de datos.

Facundo Ríos, https://orcid.org/0000-0002-6868-3480 Recolección de datos.

Guillermo Agorrody, https://orcid.org/0000-0002-92416681 Recolección de datos.

Ricardo Robaina, https://orcid.org/0000-00020363-8574 Análisis estadístico.

Víctor Dayan, https://orcid.org/0000-0002-5470-0585 Ejecución, análisis estadístico, interpretación de resultados.

Este artículo fue aceptado para su publicación por: Editor jefe Dr. Gerardo Soca.

\section{Bibliografía}

1. Issa ZF, Miller JM, Zipes DP. Clinical arrhythmology and electrophysiology: a companion to Braunwald's Heart Disease: Second Edition;2012:727p. Washignton: Elsevier;2012. 727 p. doi: 10.1016/ C2010-0-68723-2

2. Wolf PA, Abbott RD, Kannel WB. Atrial fibrillation as an independent risk factor for stroke: the Framingham Study. Stroke. 1991;22(8):983-8. doi: 10. 1161/01.str.22.8.983

3. Al-Saady NM, Obel OA, Camm AJ. Left atrial appendage: structure, function, and role in throm- 
boembolism. Heart. 1999;82(5):547-54. doi: 10. 1136/hrt.82.5.547

4. Glikson M, Wolff R, Hindricks G, Mandrola J, Camm AJ, Lip GYH, et al. EHRA/EAPCI expert consensus statement on catheter-based left atrial appendage occlusion - an update. Europace. 2019. pii: euz258. doi: 10.1093/europace/euz258

5. Badhwar V, Rankin JS, Damiano RJ Jr, Gillinov AM, Bakaeen FG, Edgerton JR, et al. The Society of Thoracic Surgeons 2017 clinical practice guidelines for the surgical treatment of atrial fibrillation. Ann Thorac Surg. 2017;103(1):329-41. doi: 10.1016/j.athoracsur.2016.10.076

6. Kirchof P, Benussi S, Kotecha D, Ahlsson A, Atar D, Casadei B, et al. 2016 ESC guidelines for the management of atrial fibrillation developed in collaboration with EACTS. Eur Heart J. 2016;37(38):2893-962. doi: 10.1093/eurheartj/ehw210

7. Lee RL, Vassallo P, Kruse J, Malaisrie SC, Rigolin V, Andrei AC, et al. A randomized, prospective pilot comparison of three atrial appendage elimination techniques: internal ligation, stapled excision, and surgical excision. J Thorac Cardiovasc Surg. 2016;152(4): 1075-80. doi: 10.1016/j.jtcvs.2016.06.009

8. Tsai Y-C, Phan K, Munkholm-Larsen S, Tian DH, La Meir M, Yan TD. Surgical left atrial appendage occlusion during cardiac surgery for patients with atrial fibrillation: a meta-analysis. Eur J Cardiothorac Surg. 2015;47(5):847-54.

doi: 10.1093/ejcts/ezu291

9. Yao X, Gersh BJ, Holmes DR Jr, Melduni RM, Johnsrud DO, Sangaralingham LR, et al. Association of surgical left atrial appendage occlusion with subsequent stroke and mortality among patients undergoing cardiac surgery. JAMA. 2018;319 (20):2116-26. doi: 10.1001/jama.2018.6024

10. Friedman DJ, Piccini JP, Wang T, Zheng J, Malaisrie SC, Holmes DR, et al. Association between left atrial appendage occlusion and readmission for thromboembolism among patients with atrial fibrillation undergoing concomitant cardiac surgery. JAMA. 2018;319(4):365-74. doi: 10.1001/jama.2017.20125 